\title{
Atmospheric deceleration and light curves of Draconid meteors and implications for the structure of cometary dust
}

\author{
J. Borovička, P. Spurný, and P. Koten
}

\begin{abstract}
Astronomical Institute of the Academy of Sciences, Fričova 298, 25165 Ondřejov Observatory, Czech Republic e-mail: borovic@asu.cas.cz
\end{abstract}

Received 21 June 2007 / Accepted 17 July 2007

\section{ABSTRACT}

\begin{abstract}
Aims. The observation of Draconid meteors was used to infer information on the structure, porosity, strength, and composition of the dust of comet 21P/Giacobini-Zinner.

Methods. Stereoscopic video and photographic observations of six faint and one bright Draconid meteors provided meteor morphologies, heights, light curves, and atmospheric decelerations. The spectrum of the bright meteor was also obtained. We developed a simple model of meteoroid ablation and fragmentation. The model assumes that cometary meteoroids are composed of constituent grains.

Results. By fitting the observed decelerations and light curves, we have found that the grain mass range was relatively narrow in all meteoroids but differed from case to case. Some meteoroids were coarse grained with grain masses $10^{-9}$ to $10^{-10} \mathrm{~kg}$, others were fine grained with grain masses one order of magnitude lower. Individual mm-sized meteoroids contained tens of thousands to almost a million grains (assuming grain density close to $3000 \mathrm{~kg} \mathrm{~m}^{-3}$ ). The meteoroids were porous aggregates of grains, having porosities of about $90 \%$ and bulk densities of $300 \mathrm{~kg} \mathrm{~m}^{-3}$. Grain separation started after the surface of the meteoroid received energy of $10^{6} \mathrm{~J} \mathrm{~m}^{-2}$. The separation continued during the first half of meteor trajectories. We call this phase erosion. The energy needed for grain erosion was 15-30× lower than the energy of vaporization. However, $30 \%$ of the largest meteoroid was resistant to thermal erosion; this part disrupted later mechanically under a very low dynamic pressure of $5 \mathrm{kPa}$. The relative abundances of $\mathrm{Na}, \mathrm{Mg}$, and Fe were nearly chondritic, but differential ablation caused preferential loss of sodium at the beginning of the trajectory.
\end{abstract}

Key words. meteors, meteoroids - comets: individual: 21P/Giacobini-Zinner

\section{Introduction}

The October Draconid meteor shower is produced by meteoroids released from the Jupiter family comet $21 \mathrm{P} /$ Giacobini-Zinner. The comet orbits the Sun with a period of 6.6 years. The meteoroids encounter terrestrial atmosphere around October 8th, five days after they have reached their perihelion at $0.995 \mathrm{AU}$, with a relatively low speed of $23 \mathrm{~km} \mathrm{~s}^{-1}$. Strong meteor activity was observed only in some of the years when the parent comet also reached perihelion, namely in 1933, 1946, 1985, and 1998 (Jenniskens 2006).

The Draconid material is used in the literature as a prototype of the most fragile meteoritic material encountering terrestrial atmosphere. Peculiar properties of Draconids were first recognized by Jacchia et al. (1950) on the basis of single-station photographs of more than 200 meteors brighter than zero magnitude, obtained during the 1946 storm. They found that Draconids appeared and disappeared at greater heights than sporadic meteors of similar speeds. Trail lengths and durations were found to be shorter by a factor of 3 in comparison with other meteors. Jacchia et al. (1950) concluded that the 1946 Draconids were composed of "softer" material, more easily melted or vaporized than that of ordinary meteors.

Peculiar properties of Draconid meteors became even more evident after two Draconids were photographed by SuperSchmidt cameras from two stations in New Mexico in 1953 (Jacchia 1956). The Super-Schmidt cameras provided still unsurprassed positional data on relatively faint meteors of about zero photographic magnitude. The data allowed the reliable measurement of atmospheric deceleration along meteor trajectories. The two Draconids showed much larger $(50-100 \times)$ deceleration than other meteors. Moreover, the shutter breaks were visible only in the first third of the trajectory; the rest of the trail was continous (so called terminal blending). The obvious explanation was severe fragmentation of the meteoroid. Differential deceleration of fragments of various sizes caused meteor elongation.

Jacchia (1955) concluded that fragmentation was a common phenomenon among all meteors photographed by SuperSchmidt cameras, not only Draconids. Shorter trajectories and progressively larger decelerations than expected for a meteoroid of a given mass, unexpectedly steep increase of meteor brightness at the beginning, and terminal blending effects were observed quite often. These data led Jacchia (1955) to the conclusion that the majority of meteoroids are porous and fragile bodies, easily breaking into fragments, in accordance with the icy-conglomerate comet model of Whipple (1951). Öpik (1955) started to call such meteoroids dustballs.

Meteoroid fragmentation can proceed in several ways. Jacchia (1955) was in favor of progressive fragmentation, i.e. fragmentation of a meteoroid into parts, which continue to crumble. Other forms are quasi-continuous fragmentation, i.e. quasicontinuous detachment of small particles from the main body, and sudden disruption into a large number of particles.

Verniani (1969), interpreting Super-Schmidt data, concluded that almost all meteoroids are porous bodies with the bulk density of about $300 \mathrm{~kg} \mathrm{~m}^{-3}$. The Draconids, however, were an 
exception, with an extremely low density estimated by Verniani (1969): $<10 \mathrm{~kg} \mathrm{~m}^{-3}$.

The results of Verniani were criticized by Ceplecha (1988). He argued that Verniani caused biases when selecting meteors for his analysis. Ceplecha (1968) clearly showed, on the basis of meteor beginning heights and decelerations, that different groups of meteoroids exist. The two main groups were designated A and C. The Draconids, however, did not fit in either of these groups and were later set as a prototype of group D (Ceplecha 1988). Ceplecha (1968) considered different material properties (such as heat capacity etc.), as well as different fragmentation ability, as the reasons of group existence. Ceplecha (1988) listed typical bulk densities for different groups: $2000 \mathrm{~kg} \mathrm{~m}^{-3}$ for group A, $750 \mathrm{~kg} \mathrm{~m}^{-3}$ for group C, and $270 \mathrm{~kg} \mathrm{~m}^{-3}$ for group D (including Draconids).

Hawkes \& Jones (1975) formed a quantitative model of ablation of dustball meteoroids. They introduced the concept of a "glue" that holds together metallic or stony grains and has a lower boiling point than the grains. The grains are released after the glue is evaporated. The authors used the results of Simonenko (1968), who found by analyzing the flares of brighter meteors, that the fundamental grain size lies in a relatively narrow range around $140 \mu \mathrm{m}$ in diameter. All grains were assumed to be of that size.

Hawkes \& Jones (1975) considered quasi-continuous grain release. Nevertheless, in their model all grains were released from small meteoroids before the grain ablation started, i.e. before the meteor beginning. Up to a certain mass-limit, the length of meteor trajectory was therefore independent of initial meteoroid mass. Observational data suggested that this is the case for meteoroids smaller than about $10^{-5} \mathrm{~kg}$. From this, Hawkes \& Jones (1975) computed the amount of energy required for meteoroid disintegration $X \approx 2 \times 10^{6} \mathrm{~J} \mathrm{~kg}^{-1}$. This energy was needed for the evaporation of the glue. The agreement with observations could also be achieved if the amount of the glue was negligible and the grains were separated after the meteoroid temperature reached about $2000 \mathrm{~K}$.

Beech (1986) applied the method of Hawkes \& Jones (1975) to the 1946 Draconids of Jacchia et al. (1950). After averaging the height data, he found that the trajectory length was constant for the Draconids up to a mass of $10^{-2} \mathrm{~kg}$. The average meteor beginning height was $97.6 \mathrm{~km}$. The corresponding value of the disruption energy, $X$, was only $\sim 10^{5} \mathrm{~J} \mathrm{~kg}^{-1}$. This would imply disruption of a $10^{-5} \mathrm{~kg}$ Draconid at a height of $114 \mathrm{~km}$.

In the last three decades, photographic observations of faint meteors have been replaced by the television technique. This technique is more sensitive and allows observations of fainter meteors. The resolution is, however, limited, which is not favorable for deceleration measurements. On the other hand, meteor light curves can be measured relatively easily. A number of authors have used the light curve shapes as evidence of meteoroid fragmentation. The light curves were found to vary greatly from meteor to meteor but on average were nearly symmetrical, while a non-fragmenting meteor should have a maximum toward the end (e.g. Murray et al. 1999; Campbell et al. 2000; Koten et al. 2004).

Beech \& Murray (2003) modeled light curves of Leonid meteors (velocity $71 \mathrm{~km} \mathrm{~s}^{-1}$ ) in terms of the dustball meteoroid model. They allowed the meteoroid to disrupt into grains with masses ranging from $10^{-10} \mathrm{~kg}$ up to $10 \%$ of the total meteoroid mass (they considered meteoroids of total mass of $10^{-6} \mathrm{~kg}$ ). A power-law mass distribution of grains was assumed. A wide range of positions of light curve maxima was obtained by varying the mass distribution index between 1.0 and 2.0.
One problem with the power-law grain mass distribution is that differential deceleration of grains should produce meteor wakes (formed by more decelerating small fragments), which are seldom observed in faint meteors (Fischer et al. 2000, and references therein). Wakes should, however, develop more easily in slow meteors, and Leonids are not favorable in this respect.

The most advanced model of dustball meteors was created by Campbell-Brown \& Koschny (2004). They modeled heating of dustball meteoroids and considered ablation before the boiling temperature was reached, using the Clausius-Clapeyron formalism. The grains were released after the melting temperature of the glue was reached. Grain mass distribution was described by either power law or Gaussian distribution, or a combination of both. Observed light curves of three Leonid meteors were fitted by the model. The glue was assumed to produce no light. Meteor deceleration was not considered in the model. The light curves were successfully fitted with the glue melting temperature of 1000-1150 K. In two cases, the grain release started after the beginning of the meteor.

In this paper we analyze in detail the data on one bright and six faint Draconid meteors observed in 2005. Our 2005 Draconid data were already presented in Koten et al. (2007, hereafter referred to as Paper I), where we reported meteor frequencies, trajectories, radiants, orbits, masses, beginning and end heights, and light curves. The light curves were described in the classic statistical way, based on the position of the maximum. Unexpectedly, we clearly observed meteor decelerations. The simultaneous observation of decelerations, light curves, and meteor morphologies provides an opportunity for revealing the structure of individual meteoroids. For this purpose, we developed a simple model of ablation of dustball meteoroids and applied it to the Draconids.

\section{The observations}

Our observation techniques were described in Paper I. We employed double-station observations with image intensified video cameras on three nights from October 6 to October 9, 2005. A higher than expected activity of the Draconid shower occurred in the evening hours on October 8 (Campbell-Brown et al. 2006, and Paper I); we observed the end of this activity between the evening twilight and about 19 UT. Unfortunately, because of poor weather at the second station (Třebíč), most meteors observed during this period were detected only in Ondřejov. There was only one video meteor (meteor number 05A08030, hereafter shortened to 30) that was observed from both stations, and for which the trajectory could be reliably determined. Nevertheless, the weather improved over the course of the night, and an additional five Draconid video meteors were observed from both stations. These five meteors were probably part of the shower background component and did not belong to the evening peak, which was caused by the meteoroids released from the comet in 1946 (Campbell-Brown et al. 2006). Since the Draconid radiant lies high in the sky in the evening and then decreases, the observed meteors have a wide range of slopes, from 61 to 26 degrees to the horizontal plane. The cameras were stationary during the night, i.e. aiming at fixed azimuth and elevation.

In addition to the video data, one bright Draconid meteor was also recorded photographically by the all-sky Autonomous Fireball Observatories of the Czech part of the European Fireball Network (see Spurný et al. 2007, for network description). The light curve of the fireball was recorded with photoelectric detectors at the fireball observatories with a sampling rate of $500 \mathrm{~Hz}$. The fireball occurred during the evening peak outside 


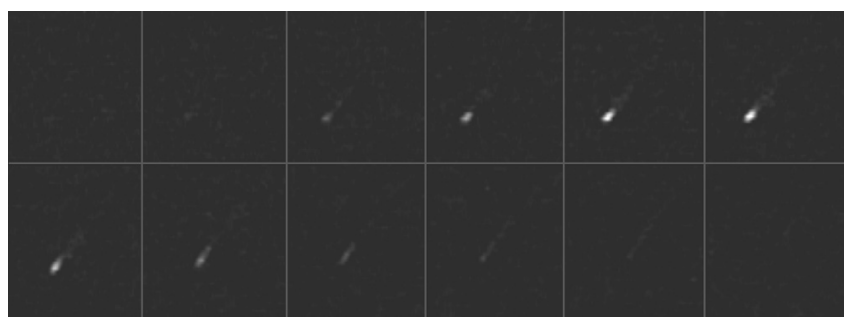

Fig. 1. Evolution of meteor 54. Parts of de-interlaced video fields centered on the meteor are shown. The background star field was subtracted. The exposure time of each image was $0.02 \mathrm{~s}$. The intervals between the shown images are $0.08 \mathrm{~s}$. The direction of meteor motion is from upper right to lower left. The time sequence starts with the upper left image and continues horizontally.

the field of view of the video cameras. Fortunately, the fireball occurred just on the opposite side of the spectral video camera from the regular field. The spectral camera, equipped with the transmission grating in front of the lens, therefore captured the fireball spectrum in the -1 st (non-blazed) spectral order. The other Draconids were too faint to produce good spectra.

\section{Meteor morphologies}

Figure 1 shows 10 images of the Draconid meteor number 54 in course of its trajectory. The elongation of the bright part of the meteor nearly corresponds to the meteor movement during the $0.02 \mathrm{~s}$ exposure time. Toward the end, however, the meteor became much more elongated and the image reveals the real shape of the radiating region. Even in the first half of the trajectory, a wake behind the meteor head was visible; the wake was, however, much fainter than the head. At the end, the whole streak, about $2 \mathrm{~km}$ long, became nearly equally bright. This behavior was typical for all Draconid meteors studied here.

Another demonstration of this effect can be seen in Fig. 2, where two Draconids are compared with one Taurid meteor. We measured the light intensity along the meteor path in each video field (with time resolution of $0.02 \mathrm{~s}$ ) in the step of $0.1 \mathrm{~km}$ in height. The resulting image for the Taurid meteor corresponds to a moving point source, except for a small effect at the end of the meteor. The Draconids, on the other hand, are blurred significantly in the final part of the trajectory, and faint wake is visible in the middle and at the beginning of the trajectory. Moreover, the time-height track of the Draconids is somewhat curved, which is an indication of meteor deceleration.

\section{Measurement of meteor images}

Positional and photometric measurements of video meteors were performed with the MetPhoto software (Koten 2002). The meteors were measured on each video frame of the PAL video record, i.e. each $0.04 \mathrm{~s}$. The meteor position was adjusted manually on a zoomed image on a computer screen. Since the moving meteor, even if it is point-like, forms a short dash during the exposure time, the leading edge of the image was measured. This does not mean adjusting the extreme edge of the signal, but rather the position, where the center of the circular image of the meteor seems to have been at the end of the exposure, and taking into account meteor brightness, the width of meteor image and the background noise. The rectangular coordinates of the meteor were then transformed into celestial coordinates using a transformation defined by the field stars. Combining data from two stations, the meteor atmospheric trajectory was determined by

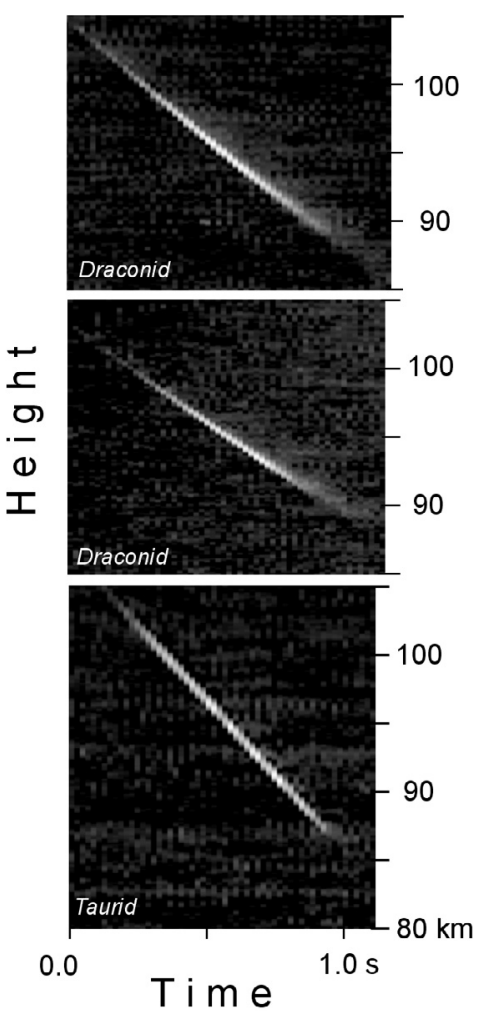

Fig. 2. Time-height intensity scans for two Draconids (05A08057 and 05A08078) and one Taurid (05A07243). Vertical scans were performed in each video field and then stacked horizontally. The background scan performed before the meteor appearance was subtracted.

the least-square method (Borovička 1990). The trajectory was assumed to be a straight line. Each measurement was then projected on the average trajectory and meteor height was computed for each time.

Meteor brightness was determined by aperture photometry on each video frame. The rectangular aperture had one axis parallel and one axis perpendicular to the direction of meteor motion. The width of the aperture was adjusted by the operator. The length of the aperture was defined by the distance travelled by the meteor during the exposure time (determined by the positional measurements on subsequent video frames) plus three pixels on each side; the extra pixels account for the spread of the signal. This procedure did not count any distant meteor wake into the signal. This approach was chosen intentionally in the software because some cameras suffer from signal persistence, and an artificial wake forms behind the meteor. The Taurid image in Fig. 2, as well as other experiments, demonstrated that the Mullard XX1332 intensifiers used in this work do not show signal persistence. Since Draconids have real wake, part of this was not counted into the meteor brightness. The modeled light curves took this effect into account by simulating the measurement procedure.

\section{Model of meteoroid sudden disruption}

We developed a simple meteor model using the concept of the dustball meteoroid. The model assumes that the meteoroid disrupts into a number of individual grains at some point, and the grains then behave as independent individual meteors. The standard drag, ablation, and radiation equations are used to describe the meteors both before and after the disruption. 


\subsection{The equations}

The drag and ablation equations are (e.g. Bronshten 1983):

$$
\begin{aligned}
m \frac{\mathrm{d} v}{\mathrm{~d} t} & =-\Gamma S \rho v^{2}, \\
\frac{\mathrm{d} m}{\mathrm{~d} t} & =-\Lambda \frac{S \rho v^{3}}{2 Q},
\end{aligned}
$$

respectively. Here $m$ is meteoroid mass, $v$ is velocity, $t$ is time, $\Gamma$ is the drag coefficient, $S$ is meteoroid cross-section, $\rho$ is density of the atmosphere, $\Lambda$ is the heat transfer coefficient, and $Q$ is the energy necessary to ablate unit mass of the meteoroid. The following quantities are usually defined (e.g. Ceplecha et al. 1998): the shape factor,

$A=S m^{-2 / 3} \delta^{2 / 3}$

the shape-density coefficient,

$K=\Gamma A \delta^{-2 / 3}=\Gamma S m^{-2 / 3}$,

and the ablation coefficient,

$\sigma=\frac{\Lambda}{2 Q \Gamma}$

Here $\delta$ is the meteoroid bulk density. Equations. (1) and (2) can be then rewritten in the form

$$
\begin{aligned}
\frac{\mathrm{d} v}{\mathrm{~d} t} & =-K m^{-1 / 3} \rho v^{2}, \\
\frac{\mathrm{d} m}{\mathrm{~d} t} & =-K \sigma m^{2 / 3} \rho v^{3} .
\end{aligned}
$$

Analytical integrals of Eqs. (6) and (7) are presented in Ceplecha et al. (1998). The solution assumes that the coefficients $K$ and $\sigma$ are constant. By providing the position (height), mass, and velocity of the meteoroid at a given time, the geometry of the trajectory (slope), the ablation coefficient, and the shape-density coefficient, one can compute the subsequent meteoroid deceleration and mass loss.

The emitted light is proportional to the mass-loss rate according to the luminosity equation (Bronshten 1983),

$$
I=-\tau \frac{v^{2}}{2} \frac{\mathrm{d} m}{\mathrm{~d} t}
$$

where $I$ is meteor luminosity in magnitude units (the meteor absolute magnitude is $M=-2.5 \log I$ ) and $\tau$ is the luminous efficiency. Note that a meteor of zero magnitude emits about $1500 \mathrm{~W}$ in the whole electromagnetic spectrum in all directions (Ceplecha et al. 1998).

The luminous efficiency is a crucial parameter here for computing initial meteoroid mass. In general, the luminous efficiency may depend on meteoroid velocity, mass, type, and the phase of the flight (Revelle \& Ceplecha 2001). For the present case, however, we considered it appropriate to use only the velocity dependence as given in Ceplecha \& McCrosky (1976). For the initial Draconid velocity of $23.5 \mathrm{~km} \mathrm{~s}^{-1}$, their value is $\tau=5 \times 10^{-6} \mathrm{~W}^{-1}$, i.e. about $0.75 \%$.

After meteoroid disruption, the whole meteoroid mass at that moment, $m_{\mathrm{f}}$, is assumed to be separated into a number of individual grains of masses in the interval $\left(m_{1}, m_{\mathrm{u}}\right)$, where $m_{\mathrm{u}}$ is the upper grain mass limit and $m_{1}$ is the lower grain mass limit. We assumed power-law mass distribution in the form

$n(m)=C m^{-s}, \quad m_{1}<m<m_{\mathrm{u}}$, where $n(m)$ is the number of grains of mass $m, s$ is the mass distribution index, and $C$ is a constant.

In our simulations, we sorted the grains into mass bins of the masses $m_{0}, m_{1}, \ldots m_{k}$, with $m_{0}$ equal to $m_{\mathrm{u}}$. The mass of all grains within the $i$ th bin was set to

$m_{i}=m_{0} p^{i}$

where the mass sorting parameter was chosen $p=10^{-0.1}=$ 0.7943 , so that there are ten mass bins per order of magnitude in mass. The number of mass bins is

$k=\log \left(m_{\mathrm{u}} / m_{1}\right) / \log p$.

Should another value of $p$ be chosen, the number of mass bins and the number of grains in each mass bin will change, but other results, e.g. the grain mass limits and the total number of grains, will remain the same. The number of grains in the $i$ th bin is

$n_{i}=n_{0}\left(\frac{m_{0}}{m_{i}}\right)^{s-1}=n_{0} p^{(1-s) i}$.

Since the total mass of grains must be equal to the meteoroid mass,

$m_{\mathrm{f}}=\sum_{i=0}^{k} n_{i} m_{i}=n_{0} m_{0} \sum_{i=0}^{k} p^{(2-s) i}$

the number of the largest grains is

$\begin{array}{lll}n_{0}=\frac{m_{\mathrm{f}}}{m_{0}(k+1)}, & \text { for } s=2, \\ n_{0}=\frac{m_{\mathrm{f}}}{m_{0}} \cdot \frac{1-p^{(2-s) k}}{1-p^{(2-s)}}, & \text { for } s \neq 2 .\end{array}$

\subsection{Free parameters}

The geometry of meteor trajectory (beginning height, trajectory slope) is known for all meteors and was given in Paper I. The initial velocity is more problematic. Because of fragmentation and subsequent deceleration, only a few measurements at the beginning of the trajectory are relevant for determination of the initial velocity. The resulting error is quite large (up to $0.5 \mathrm{~km} \mathrm{~s}^{-1}$ ). We therefore assumed that the velocity of faint meteors was the same as for the well measured photographic fireball, i.e. $23.57 \mathrm{~km} \mathrm{~s}^{-1}$. The data proved to be consistent with this value, except for meteor 128 , where we had to use $23.50 \mathrm{~km} \mathrm{~s}^{-1}$.

The initial meteoroid mass was determined by the photometric method, i.e. from the observed light curve and Eq. (8). Note that the photometric mass given in Paper I was computed under the assumption of constant meteor velocity, so the values given here are somewhat higher.

The free parameters to be determined from the deceleration, light curve shape, and meteor morphology are the ablation coefficient and shape-density coefficient for the whole meteoroid (before disruption) and for the grains, the height of disruption, the upper and lower limit of grain masses, and the grain mass distribution index.

The observed deceleration is produced by the largest grains in this model, since the largest grains are the leading ones. The deceleration does not depend directly on the mass, but on the product $K m_{\mathrm{u}}^{-1 / 3}$. The deceleration also depends on the height of disruption and on the ablation coefficient of grains (assumed to be the same for all grains). 


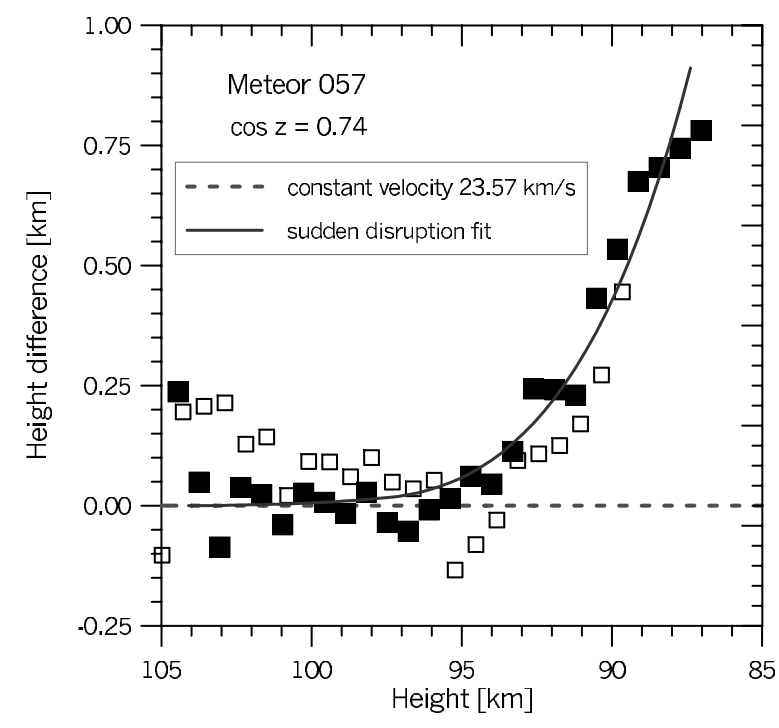

Fig. 3. Deceleration of meteor 57. Meteor height expected for the given time and constant meteor velocity of $23.57 \mathrm{~km} \mathrm{~s}^{-1}$ is given on the horizontal axis. The difference between the observed height and the expected height is given on the vertical axis. Filled squares are measurements from the primary station, empty squares come from the secondary station (more noisy). The continuous line is the fit with the sudden disruption model.

To fit the light curve, knowledge of grain masses is needed. To compute $m_{\mathrm{u}}$ from the deceleration, we assumed $K_{\mathrm{g}}=$ $0.0058 \mathrm{~m}^{2} \mathrm{~kg}^{-2 / 3}(\Gamma=1$, spherical grains with $A=1.21$, and grain density $\delta_{\mathrm{g}}=3000 \mathrm{~kg} \mathrm{~m}^{-3}$ ). The lower mass limit $m_{\mathrm{l}}$ is found from the shape of the light curve. Small grains evaporate quickly, and the smaller the grains, the larger is the light increase after the disruption. We did not take into account the grain initial heating and grain thermal radiation, which can delay the evaporation of small grains (Jones \& Kaiser 1966). These effects could refine our results, but we do not expect significant changes since the grains are released at heights where the atmosphere can heat the grains efficiently.

The mass distribution index $s$ has a relatively small effect on the shape of the light curve. Nevertheless, $s$ influences the intensity profile along the meteor. The values near 2.5 produce nearly constant brightness along the meteor streak toward the meteor end.

If the meteor was observed before the disruption, its brightness provides important information about the dustball. Since the meteoroid mass and velocity are known, the luminosity at a given height is proportional to the product $K \sigma$, as can be seen from the Eqs. (7) and (8).

\subsection{Fitting the data}

To fit the observed decelerations and light curves, the free parameters were adjusted by the trial-and-error method. Atmospheric densities were taken from CIRA (1972). The resulting fits for meteor 57 are given in Figs. 3 and 4. The deceleration could be fitted quite well. Figure 3 also shows that the deceleration was observed at both stations, though the data from the secondary station (Třebíč) show larger scatter. The light curve, however, could be fitted well only on the descending part. The modeled sudden disruption at time $0.4 \mathrm{~s}$ and a height of $97.5 \mathrm{~km}$ produced too sharp a jump on the ascending part of the light curve, which was not observed (Fig. 4). The situation

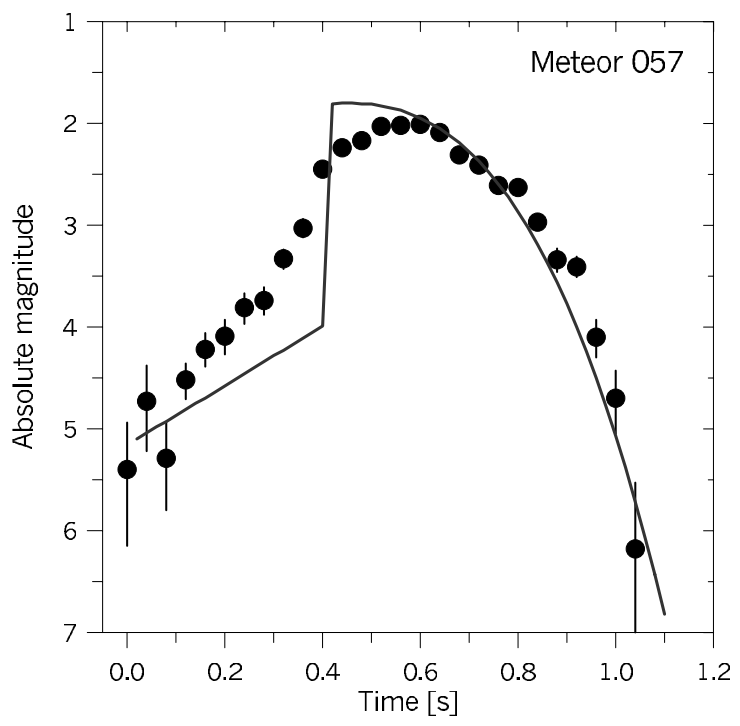

Fig. 4. Observed light curve of meteor 57 (circles) and a fit with the sudden disruption model.

was quite similar for other meteors. It is therefore clear that meteoroid disruption was not a sudden event, but a gradual process. To improve the fit we modified our model accordingly.

\section{Model of meteoroid erosion}

We assumed that instead of sudden disruption, the grains are released by gradual erosion. The erosion starts at a given point along the trajectory, and is a form of quasi-continuous fragmentation. After the erosion has started, the mass loss of the dustball meteoroid is

$\frac{\mathrm{d} m}{\mathrm{~d} t}=\left(\frac{\mathrm{d} m}{\mathrm{~d} t}\right)_{\text {ablation }}+\left(\frac{\mathrm{d} m}{\mathrm{~d} t}\right)_{\text {erosion }}$.

The first term is given by Eq. (7). For erosion, we define the erosion coefficient, $\eta$, analogous to the ablation coefficient $\sigma$. The erosion rate is then

$\left(\frac{\mathrm{d} m}{\mathrm{~d} t}\right)_{\text {erosion }}=-K \eta m^{2 / 3} \rho v^{3}$.

The eroded mass was computed from Eq. (17) in steps of $0.02 \mathrm{~s}$. The eroded mass does not immediately contribute to meteor radiation. It is distributed into individual grains according to Eq. (9) and each grain then ablates regularly. The number of grains in each mass bin was computed from Eqs. (10)-(15), with $m_{\mathrm{f}}$ equal to the eroded mass. The erosion continued until all the mass of the dustball was exhausted (except for the photographic fireballsee Sect. 6.1).

In comparison with the model of sudden disruption, the erosion model has one additional parameter - the erosion coefficient. Instead of the height of disruption there is the height of the beginning of erosion. Since the grains are released in different quantities at various heights, the distribution of brightness along the meteor streak becomes more complicated. Our procedure provided both the position of the leading edge and the position of the brightest bin of grains (a group of grains of the same mass released at the same time). The resulting fits are shown in Fig. 5 for all meteors. The brightest point in the erosion model often fits the measurements better than the sudden disruption model. 

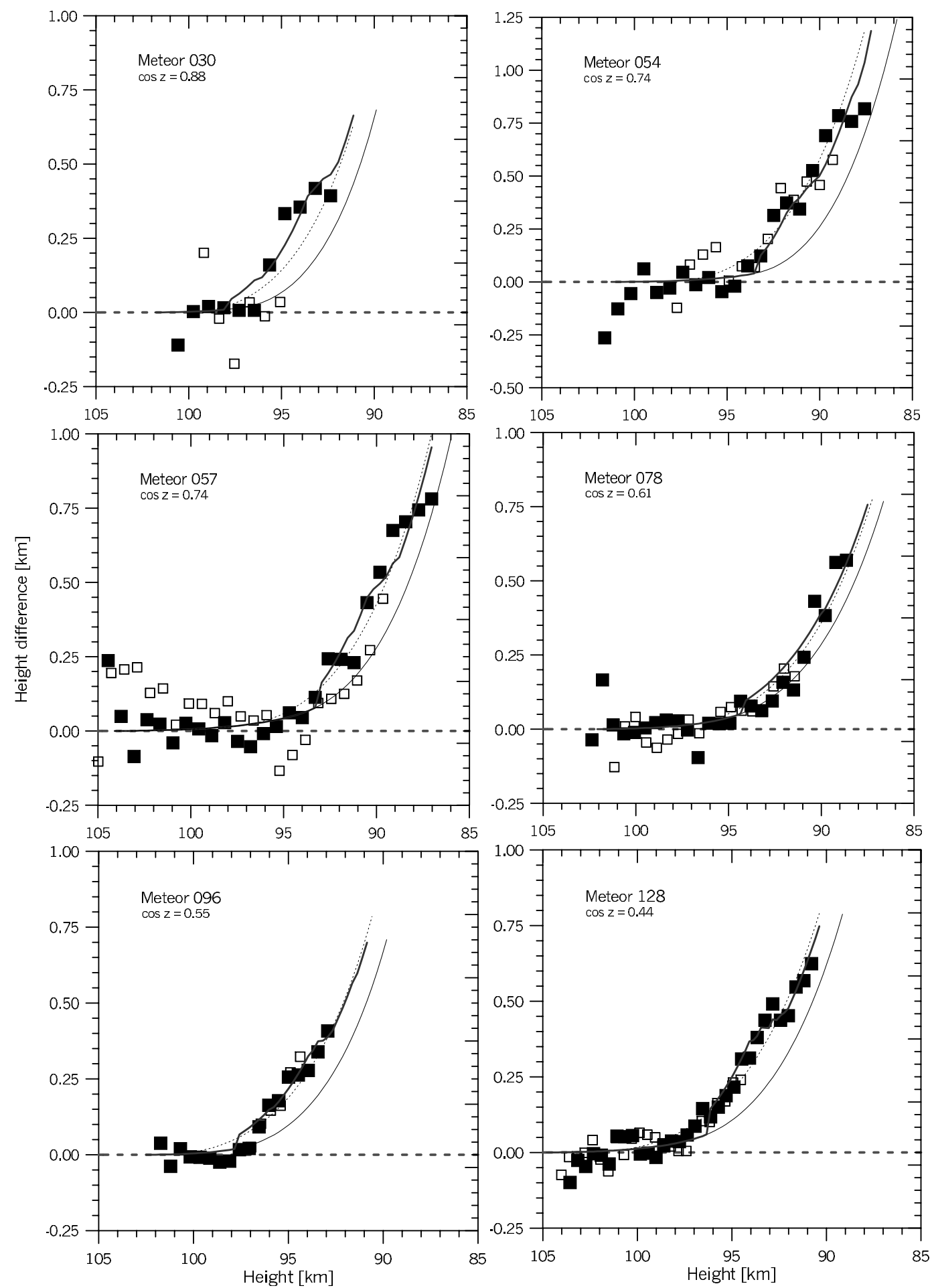

Fig. 5. Decelerations of six meteors. Three fits are shown: the brightest points in the erosion model (thick line), the leading edge in the erosion model (thin line), and the sudden disruption model fit (dotted line). For other explanation see Fig. 3.

The fits of light curves are shown in Fig. 6. Though some irregularities on the light curves could not be explained by the model, the overall shapes could be explained, although the shapes varied from meteor to meteor. The computed light curves always turn up sharply at the start of the erosion and are smooth afterwards, reaching their maxima shortly before the end of the erosion phase.

Our model also provided intensity distribution in meteors and their wakes. The result for meteor 57 is shown in Fig. 7. The prolongation of the meteor toward the end is qualitatively in agreement with observations. The modeled prolongation is, however, lower than observed. The wake in earlier parts of the trajectory is not present in the model. These discrepancies have probably been caused by early separation of very small grains, not contained in the model; however they must form a negligible part of meteoroid mass. Considering the deceleration, light curve, and main features of meteor morphology, we conclude that the agreement of the erosion model with observations is reasonably good. 

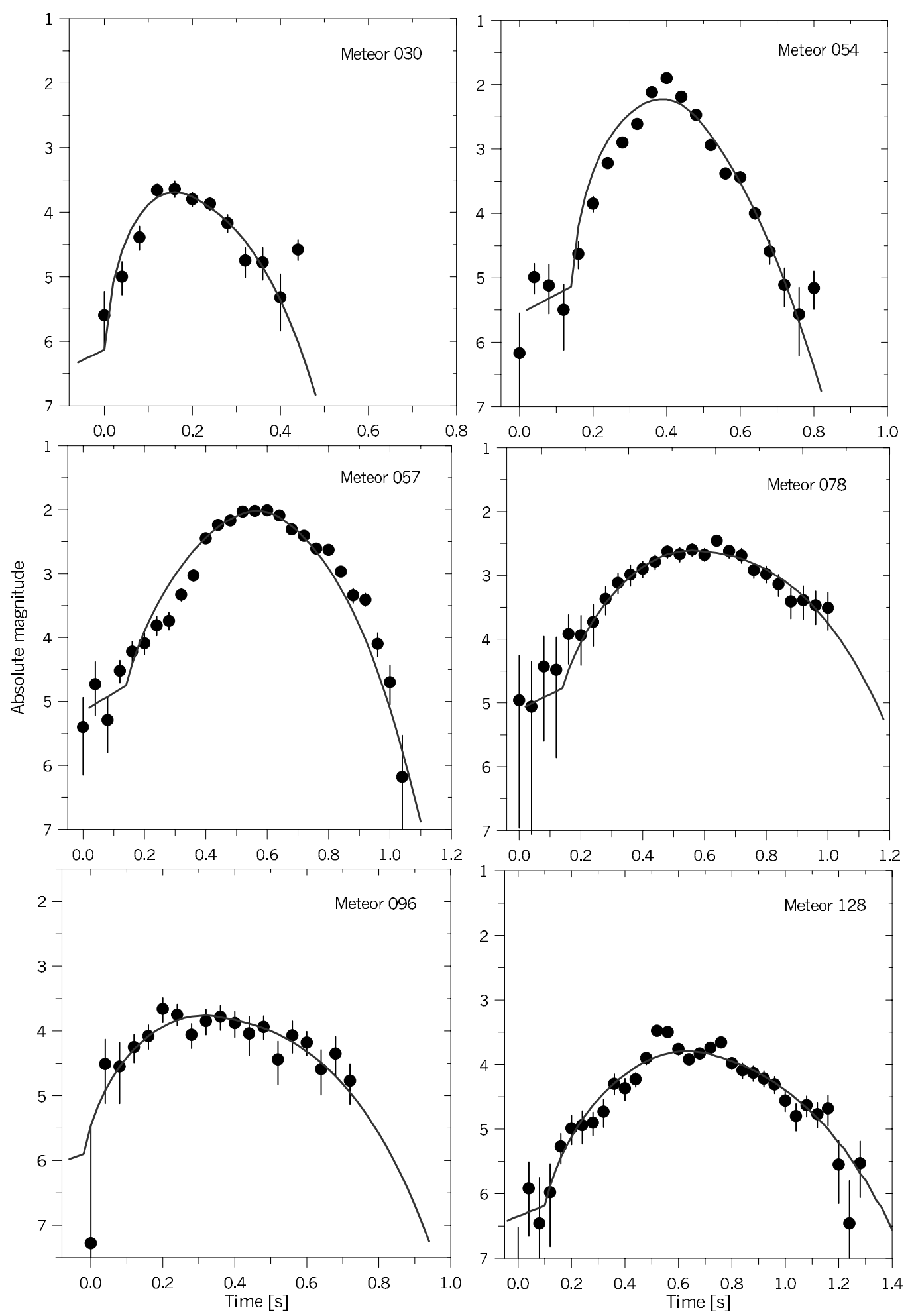

Fig. 6. Light curves of six faint Draconid meteors and the fits by the erosion model.

\subsection{The photographic fireball}

The brightest Draconid meteor of the night was captured by allsky photographic cameras and their radiometric sensors. These systems are much less sensitive than video cameras, having a limiting magnitude about -3 . The fireball showed a nearly symmetrical part of the light curve at the beginning, with a maximum of -4 mag reached at the height of $91 \mathrm{~km}$. It subsequently disappeared below the photographic limit, only to produce a brief terminal flare of $-5.5 \mathrm{mag}$ at the height of $83 \mathrm{~km}$ (Fig. 8). The fireball did not show any measurable deceleration.

The assumption that only $70 \%$ of meteoroid mass was subject to erosion was needed to model the fireball light curve successfully. The other part must have ablated regularly until the height of $83.9 \mathrm{~km}$, where it disrupted suddenly. The parameters of the grains (mass distribution, ablation coefficient) were the same for the grains released by gradual erosion and for the 


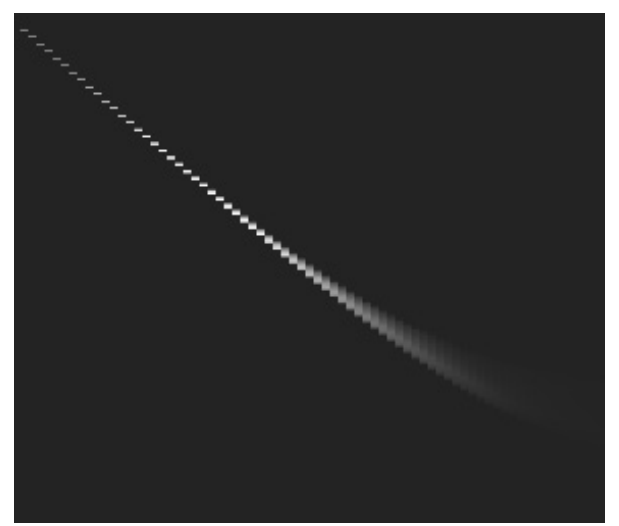

Fig. 7. Simulated time-height intensity scan for meteor 57. Compare to Fig. 2.

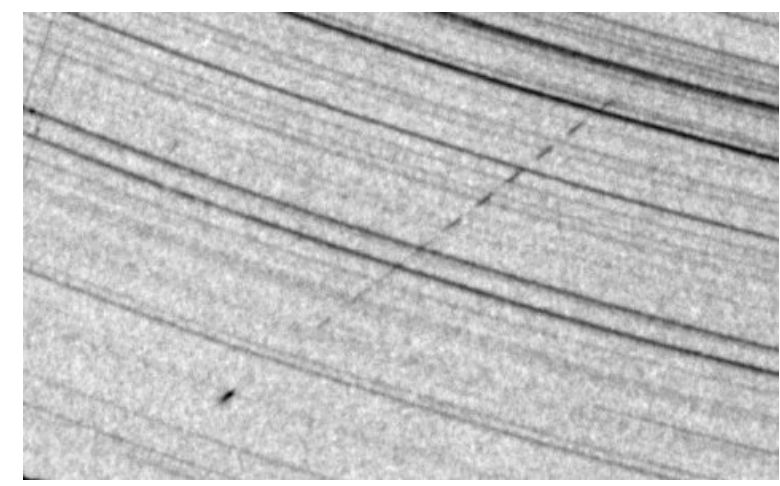

Fig. 8. Detail of all-sky photograph showing the Draconid fireball EN 081005B. The fireball flew from upper right to lower left. Rotating shutter interrupted the exposure 21 times per second. Numerous star trails are visible on more than $10 \mathrm{~h}$ long exposure.

grains released by sudden disruption at lower height. The fit of the light curve is shown in Fig. 9.

\section{Results from decelerations and light curves}

In this section we present the actual values of the parameters used for the fits in Figs. 5, 6, and 9.

\subsection{Masses and grain distribution}

Table 1 contains initial meteoroid velocity, $v_{\infty}$, trajectory slope (cosine of radiant zenith distance, $z_{\mathrm{R}}$ ), the height, $h_{0}$, at time zero (for time scales used in Figs. 4, 6, and 9), initial mass, $m_{\infty}$, the upper and lower cut-off of grain masses, $m_{\mathrm{u}}$ and $m_{\mathrm{l}}$, grain mass distribution index, $s$, grain size range, number of grains in the largest mass bin, $n_{0}$, and total number of grains, $N=\sum n_{i}$. All values were computed assuming spherical grains with a density of $3000 \mathrm{~kg} \mathrm{~m}^{-3}$, the drag coefficient equal to unity, and the luminous efficiency given in Sect. 5.1.

Note that there is no way to determine grain density from our data. Identical fits can be obtained for any grain density. If the density is multiplied by factor $f$, the grain masses must be multiplied by $f^{-2}$, grain numbers by $f^{2}$, and grain sizes by $f^{-1}$. Should the grain density be $600 \mathrm{~kg} \mathrm{~m}^{-3}$, for example, $m_{\mathrm{u}}$ and $m_{1}$ in Table 1 would be multiplied by 25 , grain sizes would be 5 times larger, and grain numbers, $n_{0}$ and $N, 25$ times lower.

If the grain density was close to $3000 \mathrm{~kg} \mathrm{~m}^{-3}$, the small meteoroids contained tens of thousands, up to almost a million,

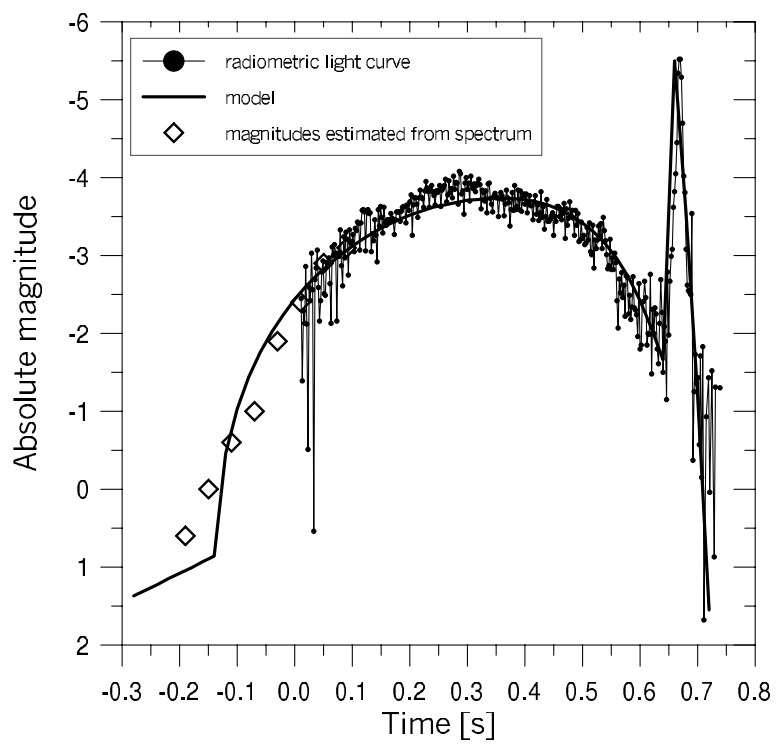

Fig. 9. Light curve of the photographic fireball as measured by the radiometric system and fitted by the model. A few data points at the beginning were supplied by roughly estimating fireball brightness from the video spectrum.

constituent grains. The grain mass range was relatively narrow in all cases, less than an order of magnitude. However, the grain masses (and sizes) were different in each meteoroid. Meteoroid 78 was coarse grained, with grain sizes around 100 microns and a relatively flat mass distribution index of 1.8. On the other hand, meteoroid 30 was fine grained, with all grains smaller than 40 microns and a mass distribution index of 2.7. Interestingly, the large photographic meteoroid was also fine grained and contained almost a billion grains. The mass distribution of grains in all meteoroids is plotted in Fig. 10.

\subsection{Physical characteristics}

Table 2 contains the derived physical characteristics of Draconid meteoroids. The ablation coefficient $\sigma$ was derived for grains from the deceleration. Nevertheless, we assumed it to be valid also for the dustball meteoroid as a whole (see the discussion below). The ablation coefficients are about $2-5 \times$ larger than the intrinsic ablation coefficients found for bright fireballs by Ceplecha \& Revelle (2005). Since large fireballs radiate at lower heights, where meteoroid shielding by vapors develops, the difference may be caused by the difference in the heat transfer coefficient $\Lambda$. The ablation energy may be nearly the same. Assuming $\Lambda=\Gamma=1$ for the Draconids, the ablation energy is $Q \approx 2 \times 10^{7} \mathrm{~J} \mathrm{~kg}^{-1}$. Note that Fischer et al. (2000) assumed $\Lambda=\Gamma=1$ and $Q=6 \times 10^{6} \mathrm{~J} \mathrm{~kg}^{-1}$, and Campbell-Brown \& Koschny (2004) assumed $\Lambda=0.5$ and $Q=3.8 \times 10^{6} \mathrm{~J} \mathrm{~kg}^{-1}$, both resulting in larger ablation coefficients than we found.

Since $\Lambda$ and $\Gamma$ are the same for ablation and erosion, the comparison of $\sigma$ and $\eta$ gives the direct comparison of the ablation and erosion energies. The erosion energy is nearly $15-30 \times$ lower than the ablation energy (Table 2). Since the erosion energy is consumed only for the separation of grains from the dustball, is is understandable that it is much lower than the energy necessary for complete vaporization. Moreover, our results suggest that the erosion energy is lower for fine-grained meteoroids.

The height at the beginning of erosion, $h_{\mathrm{es}}$, was close to $100 \mathrm{~km}$ in all cases. One may suppose that the erosion started after the meteoroid received a critical amount of energy from 
Table 1. Trajectories, masses, and grain distributions in Draconid meteoroids.

\begin{tabular}{lllllllllll}
\hline \hline $\begin{array}{l}\text { Met. } \\
\text { No. }\end{array}$ & $\begin{array}{l}v_{\infty} \\
\left(\mathrm{km} \mathrm{s}^{-1}\right)\end{array}$ & $\begin{array}{c}h_{0} \\
(\mathrm{~km})\end{array}$ & $\cos z_{\mathrm{R}}$ & $\begin{array}{l}m_{\infty} \\
(\mathrm{kg})\end{array}$ & $\begin{array}{l}m_{\mathrm{u}} \\
(\mathrm{kg})\end{array}$ & $\begin{array}{l}m_{\mathrm{l}} \\
(\mathrm{kg})\end{array}$ & $s$ & $\begin{array}{l}\text { Sizes } \\
(\mu \mathrm{m})\end{array}$ & $n_{0}$ & $N$ \\
\hline 30 & 23.57 & 100.6 & 0.88 & $7.2 \times 10^{-6}$ & $1.0 \times 10^{-10}$ & $3.0 \times 10^{-11}$ & 2.7 & $40-27$ & 7300 & 144000 \\
54 & 23.57 & 101.6 & 0.74 & $3.6 \times 10^{-5}$ & $2.0 \times 10^{-10}$ & $2.0 \times 10^{-11}$ & 2.5 & $50-23$ & 8100 & 860000 \\
57 & 23.57 & 104.5 & 0.74 & $5.9 \times 10^{-5}$ & $6.0 \times 10^{-10}$ & $1.2 \times 10^{-10}$ & 2.5 & $72-42$ & 7300 & 263000 \\
78 & 23.57 & 102.4 & 0.61 & $4.8 \times 10^{-5}$ & $2.0 \times 10^{-9}$ & $8.0 \times 10^{-10}$ & 1.8 & $108-80$ & 4900 & 36500 \\
96 & 23.57 & 101.7 & 0.55 & $1.35 \times 10^{-5}$ & $5.5 \times 10^{-10}$ & $2.8 \times 10^{-10}$ & 2.5 & $70-56$ & 4800 & 34800 \\
128 & 23.50 & 103.6 & 0.44 & $2.0 \times 10^{-5}$ & $1.0 \times 10^{-9}$ & $1.6 \times 10^{-10}$ & 2.3 & $86-46$ & 1500 & 61700 \\
Phot & 23.57 & 97.1 & 0.88 & $1.6 \times 10^{-2}$ & $1.0 \times 10^{-10}$ & $6.3 \times 10^{-12}$ & 2.5 & $40-16$ & 4000000 & 850000000 \\
\hline
\end{tabular}

Table 2. Physical characteristics of Draconid meteoroids.

\begin{tabular}{lccccccccccc}
\hline \hline $\begin{array}{l}\text { Met. } \\
\text { No. }\end{array}$ & $\begin{array}{c}\sigma \\
\left(\mathrm{s}^{2} / \mathrm{km}^{2}\right)\end{array}$ & $\begin{array}{c}\eta \\
\left(\mathrm{s}^{2} / \mathrm{km}^{2}\right)\end{array}$ & $\eta / \sigma$ & $\begin{array}{c}h_{\mathrm{es}} \\
(\mathrm{km})\end{array}$ & $\begin{array}{c}h_{\mathrm{ee}} \\
(\mathrm{km})\end{array}$ & $\begin{array}{c}E_{\mathrm{S}} \\
\left(\mathrm{J} / \mathrm{m}^{2}\right)\end{array}$ & $\begin{array}{c}E_{\mathrm{V}} \\
(\mathrm{J} / \mathrm{kg})\end{array}$ & $\begin{array}{c}K_{\infty} \\
\left(\mathrm{m}^{2} / \mathrm{kg}^{2 / 3}\right)\end{array}$ & $\begin{array}{c}S_{\infty}^{1 / 2} \\
(\mathrm{~mm})\end{array}$ & $\begin{array}{c}\delta_{\text {sphere }} \\
\left(\mathrm{kg} / \mathrm{m}^{3}\right)\end{array}$ & $\begin{array}{c}\delta_{\text {flat }} \\
\left(\mathrm{kg} / \mathrm{m}^{3}\right)\end{array}$ \\
\hline 30 & 0.030 & 0.80 & 27 & 100.6 & 96.9 & $1.1 \times 10^{6}$ & $\left(2.0 \times 10^{6}\right)$ & $(0.035)$ & $(3.5)$ & & \\
54 & 0.020 & 0.50 & 25 & 99.1 & 93.2 & $1.6 \times 10^{6}$ & $1.7 \times 10^{6}$ & 0.035 & 6 & 200 & 670 \\
57 & 0.023 & 0.33 & 14 & 102.0 & 93.0 & $1.0 \times 10^{6}$ & $1.3 \times 10^{6}$ & 0.050 & 9 & 120 & 390 \\
78 & 0.022 & 0.40 & 18 & 100.4 & 94.1 & $1.6 \times 10^{6}$ & $2.0 \times 10^{6}$ & 0.045 & 7.5 & 140 & 460 \\
96 & 0.030 & 0.60 & 20 & 102.0 & 97.1 & $1.4 \times 10^{6}$ & $\left(2.0 \times 10^{6}\right)$ & $(0.035)$ & $(4.5)$ & & \\
128 & 0.020 & 0.40 & 20 & 102.5 & 96.0 & $1.6 \times 10^{6}$ & $2.1 \times 10^{6}$ & 0.035 & 5 & 200 & 670 \\
Phot & $(0.020)$ & 0.60 & $(30)$ & 100.0 & 84.3 & $1.2 \times 10^{6}$ & $\left(0.17 \times 10^{6}\right)$ & $(0.035)$ & $(47)$ & & \\
\hline
\end{tabular}

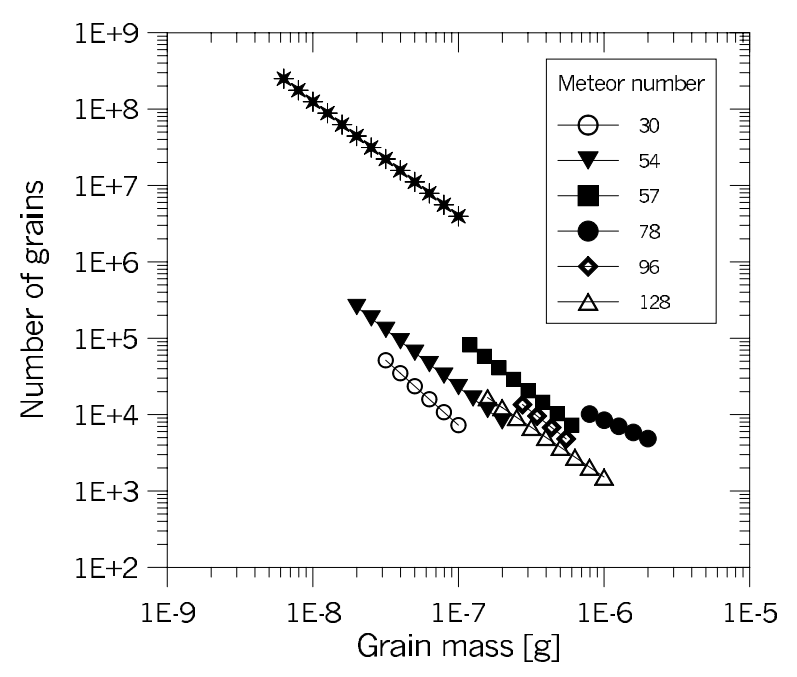

Fig. 10. Grain mass distribution in seven Draconids (assuming grain density $3000 \mathrm{~kg} \mathrm{~m}^{-3}$ ).

the incoming atmosphere. The energy received per unit crosssection, $E_{\mathrm{S}}$, can be computed considering that $\mathrm{d} E=\frac{1}{2} \Lambda S \rho v^{3} \mathrm{~d} t$ and $\mathrm{d} h=-v \cos z_{\mathrm{R}} \mathrm{d} t$ :

$E_{\mathrm{S}}=\frac{1}{2} \Lambda \frac{v^{2}}{\cos z_{\mathrm{R}}} \int_{h_{\mathrm{es}}}^{\infty} \rho(h) \mathrm{d} h$.

We assume that velocity, mass, and cross-section do not change appreciably before the start of erosion and that the meteoroid does not rotate. The energy received per unit mass, $E_{\mathrm{V}}$, is

$E_{\mathrm{V}}=E_{\mathrm{S}} \frac{S_{\infty}}{m_{\infty}}=\frac{K_{\infty} E_{\mathrm{S}}}{\Gamma m_{\infty}^{1 / 3}}$.

$E_{\mathrm{S}}$ could be computed for all meteors assuming $\Lambda=1$. To compute $E_{\mathrm{V}}$, knowledge of the shape-density coefficient of the dustball, $K_{\infty}$, is necessary. Under some assumptions this could only be evaluated for meteors observed before the start of erosion, i.e. meteors number 54, 57, 78, and 128 (see Fig. 6). Meteor brightness is directly proportional to the coefficients $K, \sigma$, and $\tau$. The same values as for the grains would give too low a meteor brightness. In principal, the dustball could have larger $\sigma$, and possibly larger $\tau$, if a volatile glue holding the grains is present. Nevertheless, we do not feel it necessary to introduce the glue without observational evidence for its presence. We therefore proceeded with the hypothesis that the observed meteor luminosity before erosion was due to a large value of $K$, i.e. low meteoroid density. The values of $E_{\mathrm{S}}, E_{\mathrm{V}}$, and $K_{\infty}$ are given in Table 2. For meteors not observed before erosion, the value $K_{\infty}=0.035 \mathrm{~m}^{2} / \mathrm{kg}^{2 / 3}$ was adopted; the real value could be lower. Uncertain values in Table 2 are given in parentheses.

The energy necessary for the start of erosion lies in a narrow range for all meteoroids if expressed per unit cross-section. If expressed per unit mass, it is lower for the largest meteoroid than for small meteoroids by an order of magnitude. Since there is no other evidence that the largest meteoroid would be so different, we conclude that the erosion starts after the surface of the meteoroid receives the energy $1.0-1.6 \times 10^{6} \mathrm{~J} \mathrm{~m}^{-2}$. The height at the end of erosion, $h_{\mathrm{ee}}$, depends on the erosion coefficient and meteoroid mass, and is also given in Table 2 . The erosion energy $Q_{\mathrm{e}} \approx 1 / 2 \eta$ lay in the range $0.6-1.5 \times 10^{6} \mathrm{~J} \mathrm{~kg}^{-1}$.

The meteoroid initial cross-section, $S_{\infty}$, was computed from $m_{\infty}$ and $K_{\infty}$ using Eq. (4) and assuming $\Gamma=1$. In Table 2 we give $S_{\infty}^{1 / 2}$, i.e. a characteristic size of the meteoroid. The video meteors were caused by meteoroids of 3-9 $\mathrm{mm}$ in size; the photographic fireball was produced by a meteoroid of several centimeters in diameter.

Should meteoroid shapes be known, meteoroid bulk densities could be computed from $K_{\infty}$ and Eq. (4). The shapes are unknown, but we made two estimates for two limiting cases: a sphere $(A=1.21)$ and a flat disk with diameter to height ratio 5:1 ( $A=2.7)$. The resulting densities for four meteoroids are given in Table 2. If the meteoroids were spherical, their densities were quite low, down to $120 \mathrm{~kg} \mathrm{~m}^{-3}$. Flat shapes would mean higher densities, provided that all meteoroids were moving with the flat side down. We estimate the truth to lie somewhere in between, 


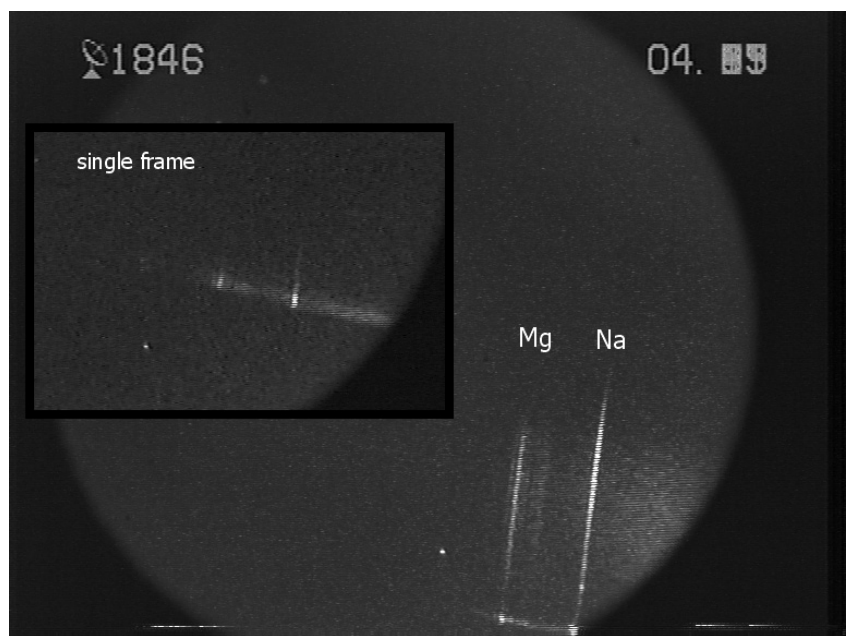

Fig. 11. Video spectrum SZ 2448 of the EN 081005B fireball. The background image was created by co-adding all frames showing the spectrum. The meteor flew from the top down and the wavelengths increase from left to right. The inset shows a single frame.

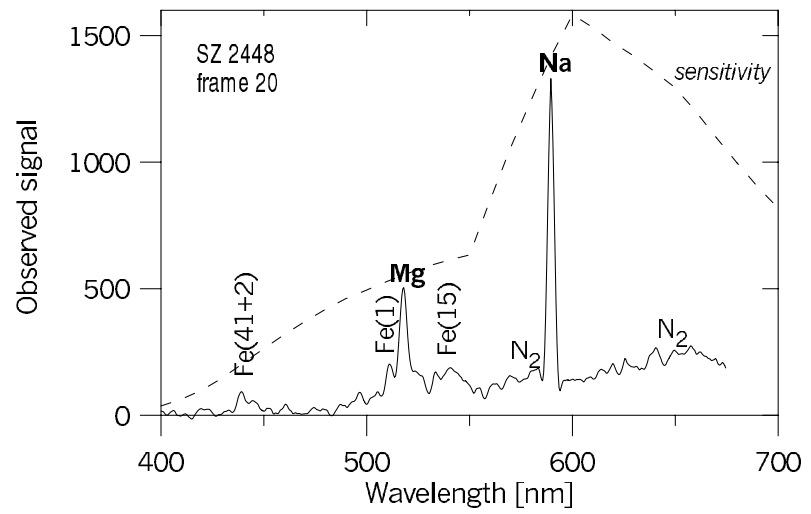

Fig. 12. Photometric tracing of a single frame of the SZ 2448 spectrum. Important emissions are identified. The numbers in the parentheses are multiplet numbers according to Moore (1945). The plotted intensities are uncalibrated. The dashed line shows relative sensitivity of the system in the -1 st order. The sensitivity is nearly two times higher for the $\mathrm{Na}$ line than for the $\mathrm{Mg}$ line.

with typical Draconid bulk density of about $300 \mathrm{~kg} \mathrm{~m}^{-3}$. The corresponding porosity is $90 \%$ (for grain density of $3000 \mathrm{~kg} \mathrm{~m}^{-3}$ ).

The terminal break-up of the photographic meteoroid was caused by process other than gradual erosion. It is likely that mechanical strength of the meteoroid was exceeded. The dynamic pressure at the moment of break-up was $P=\rho v^{2}=5 \mathrm{kPa}$.

\section{Spectral data}

The spectral camera captured four Draconid spectra; three of them are, however, very faint and of limited use. The spectra of two single station meteors show only the $\mathrm{Mg}$ line; the Na line was out of the field of view. The spectrum of meteor 57 shows both $\mathrm{Mg}$ and $\mathrm{Na}$. The $\mathrm{Na}$ line is somewhat fainter than the $\mathrm{Mg}$ line, but precise measurement was not possible. Fortunately, the spectrum of the photographic fireball was captured in the -1 st spectral order (on the non-blazed side of the grating). The spectrum is reproduced in Fig. 11 and the photometric tracing is given in Fig. 12. The two dominant emissions in the visible region are the $\mathrm{NaI}$ line at $589 \mathrm{~nm}$ and the $\mathrm{Mg}$ I line at $518 \mathrm{~nm}$. Other emissions belong to $\mathrm{Fe} I$ and the $\mathrm{N}_{2}$ molecule of

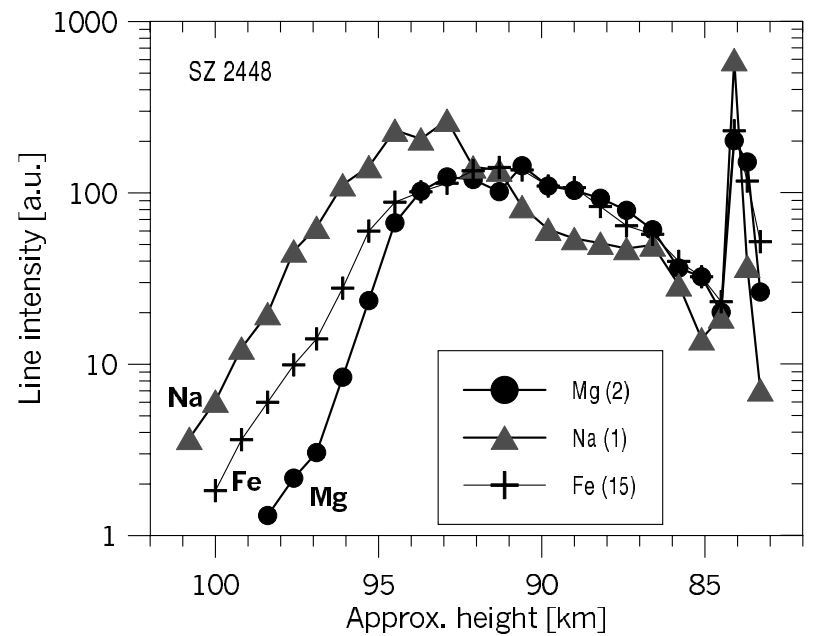

Fig. 13. Intensities of three multiplets as a function of height in the SZ2448 spectrum. The intensities have been corrected for spectral response of the instrument.

atmospheric origin. The $\mathrm{Na}$ and $\mathrm{Mg}$ lines also radiated in the short-duration train of the meteor.

The most obvious aspect of the meteor spectrum is the early start of the Na line. Figure 13 demonstrates that the Na emission started earlier than $\mathrm{Fe}$ and $\mathrm{Mg}$, and also began to fade earlier. Nevertheless, the $\mathrm{Na}$ line reappeared in full strength in the terminal flare, although the flare was shorter in $\mathrm{Na}$ than in $\mathrm{Fe}$ and $\mathrm{Mg}$. The Fe lines closely followed the Mg line, except for the beginning, where Fe was brighter.

The early appearance and disappearance of the $\mathrm{Na}$ line was observed in Leonid meteors by Borovička et al. (1999) and also in one Draconid meteor by Millman (1972). One possible explanation could be that sodium resides in the volatile glue holding the grains. The existence of the glue is, however, not necessary to produce this effect. Dust particles of about $10^{-8} \mathrm{~kg}$ and smaller are expected to ablate differentially. Theoretical computations of McNeil et al. (1998) lead to the conclusion that sodium is evaporated completely from the whole particle before magnesium starts to evaporate. Although the reality may be more complicated, the differences between the $\mathrm{Na}$ and $\mathrm{Mg}$ light curves in Fig. 13 can be understood in terms of differential grain ablation. The grains located near the surface of the porous meteoroid may lose a significant part of their sodium even before the erosion starts.

We have also tried to derive relative abundances of $\mathrm{Mg}$, $\mathrm{Na}$ and $\mathrm{Fe}$ in the meteoroid from the total radiated intensity in respective spectral lines integrated along the trajectory. The multiplet intensity ratios are $\mathrm{NaI}(1) / \mathrm{MgI}(2)=1.5$ and $\mathrm{Fe} I(15) / \mathrm{Mg} I(2)=1.1$. According to the classification of Borovička et al. (2005), the spectrum can be considered as nor$\mathrm{mal}$ in this respect. Assuming the radiating gas temperature of $4000 \mathrm{~K}$ for the meteor speed of $23 \mathrm{~km} \mathrm{~s}^{-1}$, the most probable element number ratios are $\mathrm{Na} / \mathrm{Mg}=0.064$ and $\mathrm{Fe} / \mathrm{Mg}=$ 0.66 . The uncertainty is of the order of $50 \%$. The ratios are consistent with chondritic values, and lie within the range derived by Millman (1972) for 10 photographic spectra of the 1946 Draconids (Fig. 14).

\section{Discussion}

We have applied a new approach for the analysis of atmospheric deceleration and light curves of faint Draconid meteors. 


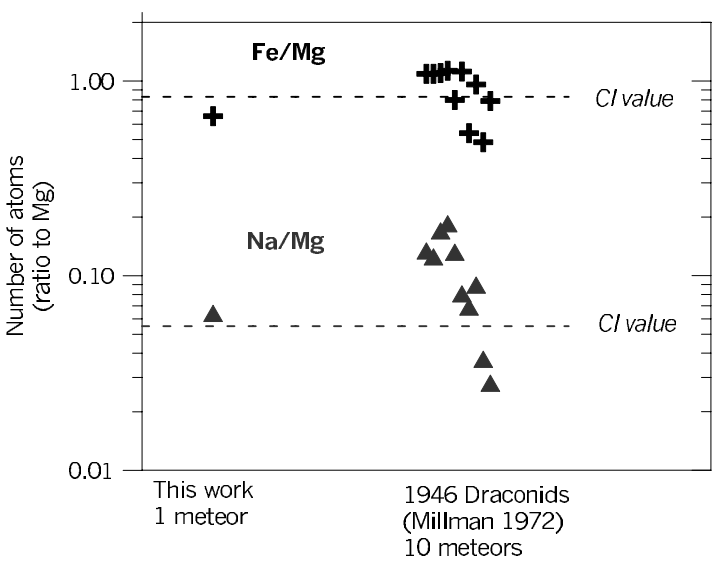

Fig. 14. $\mathrm{Fe} / \mathrm{Mg}$ and $\mathrm{Na} / \mathrm{Mg}$ ratios (by number of atoms) in the EN 081005B Draconid and 10 Draconids analyzed by Millman (1972). The dashed horizontal lines mark the values for carbonaceous chondrites.

Additional information was obtained from the analysis of a bright Draconid fireball and its spectrum. As a result, we were able to describe the structure of Draconid meteoroids and their behavior during atmospheric entry.

Our analysis revealed similar behavior to that which Campbell-Brown \& Koschny (2004) found from the analysis of Leonid light curves. Both works have shown that meteoroids disrupt gradually near the beginning of the luminous trajectories into constituent grains, and that the size range of the grains is relatively narrow. Campbell-Brown \& Koschny (2004) used mostly Gaussian mass distribution, we preferred a power law, but in a limited mass range.

Campbell-Brown \& Koschny (2004) used the concept of a volatile glue holding the grains together. In their model, the grains started to be released after the temperature of the glue reached $\sim 1100 \mathrm{~K}$. Although the presence of the glue cannot be excluded, we consider it unlikely. In our concept, dustball meteoroids are porous aggregates of constituent grains similar to the dust aggregates produced in the laboratory by Blum et al. (2006). The authors obtained $\mathrm{cm}$-sized aggregates with porosities of $85-93 \%$, using $\mu \mathrm{m}$-sized constituent grains. Our results for Draconids yield the same porosities, though the grains are larger.

What causes meteoroids to start to crumble? The dynamic pressure is extremely low at the height of $\sim 100 \mathrm{~km}$. Moreover, small meteoroids are still in the free-molecule flow regime at that height (Popova 2005), so pressure is not relevant. Energy other than mechanical energy must therefore be responsible for meteoroid destruction. One possibility is thermal energy. The grains exposed to direct air molecule impacts are heated. When they become partially melted, fragile bonds between them are destroyed. After the surface grains are lost, the mechanical balance of the irregular dustball is affected and dustball erosion continues easily.

The concept of Hawkes \& Jones (1975), that meteoroids up to a certain mass-limit are completely fragmented into individual grains before the start of the luminous trajectories, was not confirmed by our observations. In particular, the application of that concept to the Draconids by Beech (1986) gave unrealistically low fragmentation energy of $10^{5} \mathrm{~J} \mathrm{~kg}^{-1}$. According to our observations, the energy received before complete fragmentation (computed from Eqs. (18) and (19) with $h_{\mathrm{ee}}$ instead of $h_{\mathrm{es}}$ ) was from $2.5 \times 10^{6} \mathrm{~J} \mathrm{~kg}^{-1}$ (for the photographic fireball) up to $6 \times 10^{6} \mathrm{~J} \mathrm{~kg}^{-1}$.
Our estimate of Draconid density of $300 \mathrm{~kg} \mathrm{~m}^{-3}$ is in agreement with the values published for Draconid-like material by Ceplecha (1988) and Revelle (2001). Babadzhanov (2002) used a model of quasi-continuous fragmentation and obtained $400 \mathrm{~kg} \mathrm{~m}^{-3}$ for the Leonids, while he ascribed significantly larger densities to some other showers; he did not observe Draconids. Bulk densities of several hundreds of $\mathrm{kg} \mathrm{m}^{-3}$ were also measured for interplanetary dust particles (Rietmeijer 2005). However, the sizes of the IDPs were similar to the sizes of constituent grains in our case, so we observed a different type of material. On the other hand, the size of constituent grains found for Draconids is smaller than found for other meteors by Simonenko (1968).

The mechanical strength of a meteoroid can easily be found by computing the dynamic pressure at the break-up point, provided that the break-up height is known. In the past, some authors assumed that the beginning of the meteor is associated with the break-up (e.g. Öpik 1955). Recently, Trigo-Rodríguez \& Llorca (2006) assumed that the break-up point corresponds to the point of maximum brightness. Using the data of Fujiwara et al. (2001) they obtained the mean break-up height of Draconids of $98 \mathrm{~km}$ and pressure of $400 \mathrm{~Pa}$. Our analysis showed that the point of maximum brightness does not correspond to any break-up (except for the photographic fireball). The erosion started earlier, but was not caused by mechanical forces. Only the fireball flare was a consequence of mechanical break-up. The inferred strength of the meteoroid is $5 \mathrm{kPa}$. Sekanina (1985) discussed a much more massive Draconid fireball PN 39043 (maximum brightness $-11 \mathrm{mag}$ ). The fireball showed an initial maximum (probably caused by erosion), and two flares at dynamic pressures of 8.6 and $19 \mathrm{kPa}$. We can therefore conclude that the strengths of more compact parts of Draconid meteoroids are of the order of 5-20 kPa. These values are low in comparison with other meteoroids, except for certain Leonids (see the summary in Borovička 2006). The strength of Draconids is equal to the compression strength of fresh snow of densities 200$250 \mathrm{~kg} \mathrm{~m}^{-3}$ (Kronholm et al. 2003). The lower limit of tensile strength of the Draconid parent comet 21P/Giacobini-Zinner was derived by Sekanina (1985) from the rotation of the comet and found to be several hundred Pascals.

Is $21 \mathrm{P} /$ Giacobini-Zinner a typical comet? It belongs to the group of comets depleted in $\mathrm{C}_{2}, \mathrm{C}_{3}$ (A'Hearn et al. 1995), and $\mathrm{C}_{2} \mathrm{H}_{6}$ (Weaver et al. 1999; Mumma et al. 2000). Anomalous wavelength-dependence of polarization was also observed (Kiselev et al. 2000). We, however, do not think that 21P/G-Z and Draconids are exceptional. Both comet and meteor observations suggest that at least some Jupiter family comets have similar properties.

\section{Conclusions}

We analyzed atmospheric decelerations, light curves, and morphologies of seven Draconid meteors. Draconid meteoroids were found to be porous aggregates of constituent grains. Their bulk density and porosity are about $300 \mathrm{~kg} \mathrm{~m}^{-3}$ and $90 \%$, respectively. The constituent grains had relatively narrow size range in all meteoroids, but the mean grain sizes varied from meteoroid to meteoroid, from about $30 \mu \mathrm{m}$ to almost $100 \mu \mathrm{m}$. The mm-sized meteoroids contain tens of thousands to hundreds of thousands of grains (assuming grain density close to $3000 \mathrm{~kg} \mathrm{~m}^{-3}$ ).

The grains located near the surface are heated by the impact of air molecules and start to lose volatile sodium first. After receiving the energy of about $10^{6} \mathrm{~J} \mathrm{~m}^{-2}$, the surface grains separate from the meteoroid. After that, the erosion is able to 
destroy the meteoroid quickly. The meteoroid disintegration is not completed before the start of the ablation as assumed by Hawkes \& Jones (1975). The energy necessary for loss of mass in form of the grains is $15-30 \times$ lower than the ablation energy. The released grains ablate separately. The ablation coefficient is $0.020-0.030 \mathrm{~s}^{2} \mathrm{~km}^{-2}$, corresponding to the ablation energy of $\approx 2 \times 10^{7} \mathrm{~J} \mathrm{~kg}^{-1}$. Differential ablation causes preferential loss of sodium. Since the grains are small, they are significantly decelerated. Differential deceleration of grains of different masses, released at various heights, causes prolongation of the meteor.

Larger (cm-sized) meteoroids may contain more compact parts that are resistant to thermal erosion. These parts ablate regularly and contribute little to meteor luminosity at greater heights. Their mechanical strength is quite low, typically 5-20 $\mathrm{kPa}$. After the dynamic pressure exceeds this limit, the material breaks up into the constituent grains almost instantaneously. Since this happens at smaller heights, the ablation of the grains is violent and causes a short and bright meteor flare.

Draconids are an example of the most fragile cosmic material encountering the Earth. They bring information about physical properties of the comet $21 \mathrm{P} /$ Giacobini-Zinner. The observed meteoroids survived the release from the comet by gas drag and traveled through interplanetary space for at least several decades. Despite their fragility, they may still represent the strongest part of the parent comet.

Acknowledgements. The authors are indebted to Dr. Z. Ceplecha for providing his computer code for simulation of meteor deceleration and for valuable comments. This work was supported by grant No. 205/05/0543 from GAČR and grant No. B300030502 from GA AV ČR. The institutional research plan number is AV0Z10030501.

\section{References}

A'Hearn, M. F., Millis, R. L., Schleicher, D. G., Osip, D. J., \& Birch, P. V. 1995, Icarus, 118, 223

Babadzhanov, P. B. 2002, A\&A, 384, 317

Beech, M. 1986, AJ, 91, 159

Beech, M., \& Murray, I. S. 2003, MNRAS, 345, 696

Blum, J., Schräpler, R., Davidsson, B. J. R., \& Trigo-Rodríguez, J. M. 2006, ApJ, 652,1768

Borovička, J. 1990, Bull. Astr. Inst. Czechosl., 41, 391

Borovička, J. 2006, in Asteroids, Comets, and Meteors, ed. D. Lazzaro, S. Ferraz-Mello, \& J. A. Fernández, IAU Symp., 229, 249

Borovička, J., Štork, R., \& Boček, J. 1999, Meteorit. \& Planet. Sci., 34, 987

Borovička, J., Koten, P., Spurný, P., Boček, J., \& Štork, R. 2005, Icarus, 174, 15

Bronshten, V. A. 1983, Physics of Meteoric Phenomena (Dordrecht: Reidel), 356

Campbell, M. D., Brown, P. G., LeBlanc, A. G., et al. 2000, Meteorit. \& Planet. Sci., 35, 1259
Campbell-Brown, M. D., \& Koschny, D. 2004, A\&A, 418, 751

Campbell-Brown, M. D., Vaubaillon, J., Brown, P., Weryk, R. J., \& Arlt, R. 2006, A\&A, 451, 339

Ceplecha, Z. 1968, Smithson. Astrophys. Obs. Spec. Rep., 279

Ceplecha, Z. 1988, Bull. Astr. Inst. Czechosl., 39, 221

Ceplecha, Z., \& McCrosky, R. E. 1976, J. Geophys. Res., 81, 6257

Ceplecha, Z., \& Revelle, D. O. 2005, Meteorit. \& Planet. Sci., 40, 35

Ceplecha, Z., Borovička, J., Elford, W. G., et al. 1998, Space Sci. Rev., 84, 327

CIRA 1972: COSPAR International Reference Atmosphere 1972 (Berlin: Akademie-Verlag)

Fischer, A. A., Hawkes, R. L., Murray, I. S., Campbell, M. D., \& LeBlanc, A. G. 2000, Planet. Space Sci., 48, 911

Fujiwara, Y., Ueda, M., Sugimoto, M., et al. 2001, in Proc. Meteoroids 2001 Conf., ed. B. Warmbein, Kiruna, Sweden, ESA-SP, 495, 123

Hawkes, R. L., \& Jones, J. 1975, MNRAS, 173, 339

Jacchia, L. G. 1955, ApJ, 121, 521

Jacchia, L. G. 1956, AJ, 61, 6

Jacchia, L. G., Kopal, Z., \& Millman, P. M. 1950, ApJ, 111, 104

Jenniskens, P. 2006, Meteor Showers and their Parent Comets (Cambridge Univ. Press), 790

Jones, J., \& Kaiser, T. R. 1966, MNRAS, 133, 411

Kiselev, N. N., Jockers, K., Rosenbush, V. K., et al. 2000, Planet. Space Sci., 48, 1005

Kronholm, K., Johnson, J. B., \& Schneebeli, M. 2003, The 3rd Inter. Conf. on Mars Polar Sci. and Exploration, abstract \#8084

Koten, P. 2002, in Proc. of the Asteroids, Comets, Meteors 2002 Conf., ed. B. Warmbein, Berlin, ESA SP, 500, 197

Koten, P., Borovička, J., Spurný, P., Betlem, H., \& Evans, S. 2004, A\&A, 428, 683

Koten, P., Borovička, J., Spurný, P., \& Štork, R. 2007, A\&A, 466, 729 (Paper I)

McNeil, W. J., Lai, S. L., \& Murad, E. 1998, J. Geophys. Res., 103 (D9), 10889

Millman, P. M. 1972, JRASC, 66, 201

Moore, C. E. 1945, Contrib. Princeton Univ. Obs., 20

Mumma, M. J., DiSanti, M. A., Russo, N. D., Magee-Sauer, K., \& Rettig, T. W. 2000, ApJ, 531, L155

Murray, I. S., Hawkes, R. L., \& Jenniskens, P. 1999, Meteorit. \& Planet. Sci., 34, 949

Öpik, E. J. 1955, Ir. Astron. J., 3, 165

Popova, O. 2005, Earth, Moon, Planets, 95, 303

Revelle, D. O. 2001, in Proc. Meteoroids 2001 Conf., ed. B. Warmbein, Kiruna, Sweden, ESA-SP, 495, 513

Revelle, D. O., \& Ceplecha, Z. 2001, in Proc. Meteoroids 2001 Conf., ed. B. Warmbein, Kiruna, Sweden, ESA-SP, 495, 507

Rietmeijer, F. J. M. 2005, Earth, Moon, Planets, 95, 321

Sekanina, Z. 1985, AJ, 90, 827

Simonenko, A. N., 1968, in Physics and Dynamics of Meteors, ed. L'. Kresák, \& P. M. Millman, IAU Symp., 33, 207

Spurný, P., Borovička, J., \& Shrbený, L. 2007, in Near Earth Objects, our Celestial Neighbors: Opportunity and Risk, ed. G. B. Valsecchi, \& D. Vokrouhlický, IAU Symp., 236, 121

Trigo-Rodríguez, J. M., \& Llorca, J. 2006, MNRAS, 372, 655. Erratum: MNRAS, 375, 415

Verniani, F. 1969, Space Sci. Rev., 10, 230

Weaver, H. A., Chin, G., Bockelée-Morvan, D., et al. 1999, Icarus, 142, 482

Whipple, F. L. 1951, ApJ, 113, 464 